\title{
Primary Idiopathic Myelofibrosis: Clinico-Epidemiological Profile and Risk Stratification in Pakistani Patients
}

\author{
Sadia Sultan*, Syed Mohammed Irfan
}

\begin{abstract}
Background: Primary idiopathic myelofibrosis (PMF) is a clonal Philadelphia chromosome-negative myeloproliferative neoplasm characterized by extramedullary hematopoiesis and marrow fibrosis. It is an uncommon hematopoietic malignancy which primarily affects elderly individuals. The rational of this study was to determine its clinico-epidemiological profile along with risk stratification in Pakistani patients. Materials and Methods: In this retrospective cross sectional study, 20 patients with idiopathic myelofibrosis were enrolled from

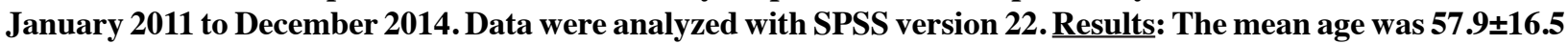
years with $70 \%$ of patients aged above 50 . The male to female ratio was $3: 1$. Overall only $10 \%$ of patients were asymptomatic and the remainder presented with constitutional symptoms. In symptomatic patients, major complaints were weakness $(80 \%)$, weight loss $(75 \%)$, abdominal discomfort $(60 \%)$, night sweats $(13 \%)$, pruritus $(5 \%)$ and cardiovascular accidents $(5 \%)$. Physical examination revealed splenomegaly as a predominant finding detected in 17 patients $(85 \%)$ with the mean splenic span of $22.2 \pm 2.04 \mathrm{~cm}$. The mean hemoglobin was $9.16 \pm 2.52$ $\mathrm{g} / \mathrm{dl}$ with the mean MCV of $88.2 \pm 19.7 \mathrm{fl}$. The total leukocyte count of $17.6 \pm 19.2 \times 10^{9} / \mathrm{l}$ and platelets count were $346.5 \pm 321.9 \times 10^{9} / 1$. Serum lactate dehydrogenase, serum creatinine and uric acid were $731.0 \pm 154.1,0.82 \pm 0.22$ and 4.76 \pm 1.33 respectively. According to risk stratification, $35 \%$ were in high risk, $40 \%$ in intermediate risk and $25 \%$ in low risk groups. Conclusions: The majority of PMF patients were male and presented with constitutional symptoms in our setting. Risk stratification revealed predominance of advanced disease in our series.
\end{abstract}

Keywords: Clinico-epidemiological profile - primary idiopathic myelofibrosis - risk stratification

Asian Pac J Cancer Prev, 16 (18), 8629-8631

\section{Introduction}

Myeloproliferative neoplasms (MPN) are a group of diseases which are characterized by excessive production of one or more myeloid lineage cells arising from genetic mutations in multipotential hematopoietic stem cells in bone marrow (Sag et al., 2015; Yang et al., 2015). Idiopathic Primary myelofibrosis (PMF) is a heterogenous Philadelphia chromosome-negative MPN exemplified by cytopenias, progressive marrow fibrosis; extramedullary hematopoiesis associated with excessive production of inflammatory cytokines and shortened survival.

The estimated incidence of PMF is $0.5-1.5$ per 100 , 000 populations (Mesa et al., 1999; Tefferi., 2000). It occurs most commonly in the sixth to seventh decade of life and both sexes are nearly equally affected (Thiele et al., 2008). The median age at diagnosis is around 66 years (Mitra et al., 2013).

Organomegaly owing to extramedullary hematopoiesis typically accompanied by symptoms of early satiety, left upper quadrant pain and abdominal distension. PMFrelated constitutional symptoms which include fatigue, pruritus, night sweats and fever have been associated with elevated inflammatory cytokines have a profound impact on patient quality of life. The QOL of patient's with PMF is virtually similar to those with advanced solid malignancies (Kiladjian et al., 2012). Furthermore, PMF can sometimes leads to debilitating complications such as thromboembolism, pulmonary hypertension and portal hypertension which may manifest at any time during the disease course (Abu-Hilal and Tawaker, 2009).

Based on the presence of specific prognostic factors, patients are broadly classified as in low, intermediate or high risk disease groups. Median overall survival has been reported to be approximately 6 years (Mitra et al., 2013). According to the Lille Prognostic Scoring System for PMF, patients diagnosed with low-risk MF may survive for 93 months or longer, whereas the median life expectancy for intermediate and high-risk patients are only 26 and 13 months, respectively (Campbell and Green, 2011).

Since to out knowledge there is no study available from Pakistan; we sought here to detail findings for a series of PMF patients. The present study is a single institution retrospective analysis of 20 patients with PMF, focusing on their clinical presentation, laboratory findings and risk stratification. 


\section{Materials and Methods}

This retrospective cross sectional study, extended from January 2011 to December 2014. During the study period, 20 patients diagnosed to have primary idiopathic myelofibrosis were enrolled in the present study. An informed consent was obtained from all the participating patients.

Patients were diagnosed to have PMF according to the World Health Organization (WHO) criteria (Thiele et al., 2008). Diagnosis required meeting all 3 major criteria and any 2 minor criteria.

\section{Major criteria includes}

Presence of megakaryocyte proliferation and atypia, accompanied by fibrosis, or in the absence of significant fibrosis, the megakaryocyte changes must be accompanied by an increased bone marrow cellularity.

Not meeting WHO criteria for other myeloid neoplasm.

Demonstration of JAK2 V617F or in the absence of a clonal marker, no evidence of bone marrow fibrosis due to underlying inflammatory or other diseases.

\section{Minor criteria}

i) Leukoerythroblastosis. ii) Increase in serum lactate dehydrogenase level. iii) Anemia. iv) Splenomegaly

Demographical data including age, gender and medical history were recorded. Hematological parameters were determined by Cell Dyne Ruby (Abott, Diagnostics). All peripheral smears were reviewed by expert hematopathologists. Serum creatinine, lactate dehydrogenase (LDH) and serum uric acid were detected by HITACHI 912 (Japan) by photometric assay. JAK2 V617F mutational analyses were done by Polymerase chain reaction (PCR). Bone marrow aspirate and trephine biopsy specimen were taken with Jamshidi needle.

Patients were stratified according to Lille Prognostic Scoring System for PMF which categorizes patients into three risk groups based on the hemoglobin level and leukocyte count at diagnosis (Campbell and Green, 2011). The two adverse prognostic factors, namely $\mathrm{Hb}<10 \mathrm{~g} / \mathrm{dL}$ and $\mathrm{WBC}<4$ or $>30 \times 10^{3} / \mathrm{L}$, was able to separate patients in three groups; with low ( 0 factor), intermediate ( 1 factor) and high (2 factors) (Benites et al., 2013).

The ethical endorsement of the study was granted by research and ethical committee $\mathrm{LNH}$ taken prior to the study.

\section{Data analysis}

Data was compiled and analyzed using SPSS version 22 . The results were expressed as mean $\pm \mathrm{SD}$ for quantitative variables and qualitative variables are presented as frequency \& percentages.

\section{Results}

During the study period, 20 patients were diagnosed as Primary idiopathic myelofibrosis.

Out of 20 patients, 15 were males (75\%) and 5 were females (25\%) with male to female ratio of 3:1. The mean age was $57.90 \pm 16.51$ years (range 22-87) with the median age of 60 years. Overall, 14 (70\%) patients were above 50 years; while $6(30 \%)$ patients were under 50 years of age. Mostly patients (90\%) were symptomatic and presented with constitutional symptoms, only $10 \%$ were asymptomatic. In symptomatic patients, major complaints were weakness $(80 \%)$; weight loss $(75 \%)$; abdominal discomfort (60\%); night sweats (13\%); cardiovascular accident $(5 \%)$ and pruritus (5\%).

Physical examination revealed splenomegaly as predominant finding detected in 17 patients $(85 \%)$ with the mean splenic span of $22.17 \pm 2.04 \mathrm{~cm}$. The hepatomegaly was detected in $11(55 \%)$ patients followed by pallor in $7(35 \%)$ patients.

The mean hemoglobin was $9.16 \pm 2.52 \mathrm{~g} / \mathrm{dl}$ with the mean MCV of $88.17 \pm 19.69 \mathrm{fl}$. The total leukocyte count of $17.63 \pm 19.17 \times 109 / 1$ and platelets count were $346.50 \pm 321.93 \times 10^{9} / 1$. Overall $13(65 \%)$ patients were anemic and $4(20 \%)$ patients had thrombocytopenia. Leucopenia and leukocytosis were seen in $5(25 \%)$ and 4 (20\%) cases respectively.

Serum lactate dehydrogenase, serum creatinine and uric acid were $731.0 \pm 154.1,0.82 \pm 0.22$ and $4.76 \pm 1.33$ respectively. According to risk stratification, $35 \%$ were in high risk; $40 \%$ in intermediate risk while $25 \%$ in low risk group.

\section{Discussion}

Primary Myelofibrosis is a clonal myeloproliferative neoplasm, in which the proliferation of an abnormal clone of hematopoietic stem cells results in marrow fibrosis. $\mathrm{PMF}$ is associated with a worse prognosis in comparison with other classic BCR/ABL-negative myeloproliferative neoplasms, which may eventually culminate into acute leukemia (Cervantes et al., 2008; Devos et al., 2015). Yet survival may range from a few months to an excess of a decade.

This hematological disease is very uncommon world widely. The present study has illustrated clinical features, hematological markers, biochemical profile and risk stratification in Pakistani PMF patients. This is a first preliminary report from our part of the world.

PMF usually develops slowly and is mainly observed in people over the age of 50 years. Most of the patients in our study presented in the $5^{\text {th }}$ to 6 th decade of life. However a large regional study reported by Sazawal et al from India, revealed PMF in relatively younger age group, with the mean age of 47 years (Sazawal et al., 2010). When compared with earlier international reports, our results are in concurrence with studies published from Germany and Sweden, where the median age were 57 and 55 years respectively (Abelsson et al., 2012; Alchalby et al., 2012).

The male gender dominance was seen in the present study which is in concurrence with the prior Indian and Thailand studies (Sazawal et al., 2010; Duangnapasatit et al., 2015). In conflict to this, international data suggest equal gender distribution (Thiele et al., 2008). This difference could be attributed to ethnic and genetic variations but needs validation in larger population based 
studies from our region.

The clinical manifestations of PMF are heterogeneous, patients are usually symptomatic. Patients often have symptoms of anemia, splenomegaly or in later stages, have general malaise, weight loss, fever or splenic infarction. As most of our patients had constitutional symptoms $(90 \%)$, this is more or less similar to studies reported from Spain (70\%) and Thailand (83.3\%) (Cervantes., 2014; Duangnapasatit et al., 2015).

Splenomegaly and hepatomegaly occurs in a significant proportion of patients. Previously one study reported from India disclosed splenomegaly in $78 \%$ of their patients (Sazawal et al., 2010). Another Thai series, also reported splenomegaly and hepatomegaly in $100 \%$ and $50 \%$ respectively which is similar to our findings of $85 \%$ and $55 \%$ respectively (Duangnapasatit et al., 2015).

Bone marrow failure eventually occurs, with consequent anemia and thrombocytopenia. Anemia is typically present and usually increases over time, being associated with poorer prognosis, advanced stage and increased morbidity. In the present study, relatively high prevalence $(65 \%)$ was detected and it is analogous with the results of two large Italian studies; $63 \%$ and $65 \%$ respectively (Marchetti et al., 2004; Vannucchi., 2011). The mean hemoglobin in our series is $9.1 \mathrm{gm} / \mathrm{dl}$. In concurrence to our results regional Thai study $(8.2 \mathrm{gm} /$ dl) revealed more or less similar findings (Duangnapasatit et al., 2015).

WBC counts in PMF are usually increased but are highly variable; a low WBC count tends to indicate a poor prognosis, seen in $25 \%$ of patients in our series. Similarly Marchetti reported leucopenia in $21 \%$ of their patients (Marchetti et al., 2004).

The risk stratification is useful to delineate the disease extent, to predict the likelihood of disease complications and most importantly to ascertain the disease prognosis. In one prior Brazilian study; $73 \%$ had low risk; intermediate in $18 \%$ and high risk disease in only $7 \%$ (Benites et al., 2013). Compared with this majority of our patients were in intermediate risk which is in consensus with study reported by German group; $56 \%$ and $23 \%$ in intermediate and high risk respectively (Alchalby et al., 2012). This may be accredited to the aggressive disease course along with concomitant delay in seeking medical consultation.

In conclusion, the study provides insight into the demographic and clinico-epidemiological characteristics of the Pakistani myelofibrotic population. Predominantly patients are symptomatic having constitutional symptoms. Presenting features are similar as reported in literature, and most of the patients had intermediate risk disease. Prospective studies should be pursueded on large patient series to investigate further disease spectrum and should incorporate novel molecular tools.

\section{Acknowledgements}

We would like to express our special thanks of $\mathrm{Mr}$. Danish Hussain for statistical analysis for this study.

\section{References}

Abelsson J, Merup M, Birgegård G, et al; Nordic MPD Study Group (2012). The outcome of allo-HSCT for 92 patients with myelofibrosis in the Nordic countries. Bone Marrow Transplant, 47, 380-6.

Abu-Hilal M, Tawaker J (2009). Portal hypertension secondary to myelofibrosis with myeloid metaplasia: a study of 13 cases.World J Gastroenterol, 15, 3128-33.

Alchalby H, Yunus DR, Zabelina T, et al (2012). Risk models predicting survival after reduced-intensity transplantation for myelofibrosis. Br J Haematol, 157, 75-85.

Benites BD, Lima CS, Lorand-Metze I, et al (2013). Primary myelofibrosis: risk stratification by IPSS identifies patients with poor clinical outcome. Clinics Sao Paulo, 68, 339-43.

Campbell PJ, Green AR (2011). Myeloproliferative neoplasms. In: Hoffbrand AV, Catovsky D, Tuddenham EG, Green AR. Post graduate hematology. Victor Hoffbrand, $6^{\text {th }}$ edition, 701 .

Cervantes F, Passamonti F, Barosi G (2008). Life expectancy and prognostic factors in the classic BCR/ABL-negative myeloproliferative disorders. Leukemia, 22, 905-14.

Cervantes F (2014). How I treat myelofibrosis. Blood, 124, 2635-42.

Devos T, Zachée P, Bron D, et al (2015). Myelofibrosis patients in Belgium: disease characteristics. Acta Clin Belg, 70, 105-11.

Duangnapasatit B, Rattarittamrong E, Rattanathammethee T, et al (2015). Clinical Manifestations and Risk Factors for Complications of Philadelphia Chromosome-Negative Myeloproliferative Neoplasms. Asian Pac J Cancer Prev, 16, 5013-8.

Kiladjian JJ, Gisslinger H, Passamonti F, et al (2012). Healthrelated quality of life (HRQoL) and symptom burden in patients (Pts) with myelofibrosis (MF) in the COMFORT-II study. J Clin Oncol, 30, 6626.

Marchetti M, Barosi G, Balestri F, et al (2004). Low-dose thalidomide ameliorates cytopenias and splenomegaly in myelofibrosis with myeloid metaplasia: a phase II trial. $J$ Clin Oncol, 22, 424-31.

Mesa RA, Silverstein MN, Jacobsen SJ, Wollan PC, Tefferi A (1999). Population-based incidence and survival figures in essential thrombocythemia and agnogenic myeloid metaplasia: an Olmsted County Study, 1976-1995. Am J Hematol, 61, 10-15.

Mitra D, Kaye JA, Piecoro LT, et al (2013). Symptom burden and splenomegaly in patients with myelofibrosis in the United States: a retrospective medical record review. Cancer Med, 2, 889-98.

Sag SO, Gorukmez O, Ture M, et al (2015). MMP2 gene-735 C/T and MMP9 gene $-1562 \mathrm{C} / \mathrm{T}$ polymorphisms in JAK2V617F positive myeloproliferative disorders. Asian Pac J Cancer Prev, 16, 443-9.

Sazawal S, Bajaj J, Chikkara S, et al (2010). Prevalence of JAK2 V617F mutation in Indian patients with chronic myeloproliferative disorders. Indian J Med Re, 132, 423-7.

Tefferi A (2000). Myelofibrosis with myeloid metaplasia. N Engl $J$ Med, 342, 1255-65.

Thiele J, Kvasnicka HM, Orazi G, Tefferi A, Vardiman JM. Primary myelofibrosis. In: Swerdlow HS, Campo E, Haris LN, et al (2008). WHO classification of tumours of haemopoietic and lymphoid tissues. Lyon: International agency for research on cancer, 44.

Vannucchi AM (2011). Management of myelofibrosis. Hematology Am Soc Hematol Educ Program. 2011, 222-30.

Yang JJ, Chen H, Zheng XQ, et al (2015). Methylated alteration of SHP1 complements mutation of JAK2 tyrosine kinase in patients with myeloproliferative neoplasm. Asian Pac J Cancer Prev, 16, 2219-25. 